\title{
Determining the Optimal Placement of Sensors on a Concrete Arch Dam Using a Quantum Genetic Algorithm
}

\author{
Kai Zhu, ${ }^{1,2,3}$ Chongshi Gu, ${ }^{1,2,3}$ Jianchun Qiu, ${ }^{1,2,3}$ Wanxin Liu, ${ }^{1,2,3}$ Chunhui Fang, ${ }^{4}$ and Bo Li ${ }^{5}$ \\ ${ }^{1}$ State Key Laboratory of Hydrology-Water Resources and Hydraulic Engineering, Hohai University, Nanjing 210098, China \\ ${ }^{2}$ National Engineering Research Center of Water Resources Efficient Utilization and Engineering Safety, Hohai University, \\ Nanjing 210098, China \\ ${ }^{3}$ College of Water-Conservancy and Hydropower, Hohai University, Nanjing 210098, China \\ ${ }^{4}$ Zhejiang Institute of Hydraulics and Estuary, Hangzhou 310020, China \\ ${ }^{5}$ Engineering Safety and Disaster Prevention Department, Changjiang River Scientific Research Institute, Wuhan 430010, China
}

Correspondence should be addressed to Kai Zhu; 879058923@qq.com

Received 18 September 2015; Accepted 1 December 2015

Academic Editor: Kourosh Kalantar-Zadeh

Copyright @ 2016 Kai Zhu et al. This is an open access article distributed under the Creative Commons Attribution License, which permits unrestricted use, distribution, and reproduction in any medium, provided the original work is properly cited.

\begin{abstract}
Structural modal identification has become increasingly important in health monitoring, fault diagnosis, vibration control, and dynamic analysis of engineering structures in recent years. Based on an analysis of traditional optimization algorithms, this paper proposes a novel sensor optimization criterion that combines the effective independence (EFI) method with the modal strain energy (MSE) method. Considering the complex structure and enormous degrees of freedom (DOFs) of modern concrete arch dam, a quantum genetic algorithm (QGA) is used to optimize the corresponding sensor network on the upstream surface of a dam. Finally, this study uses a specific concrete arch dam as an example and determines the optimal sensor placement using the proposed method. By comparing the results with the traditional optimization methods, the proposed method is shown to maximize the spatial intersection angle among the modal vectors of sensor network and can effectively resist ambient perturbations, which will make the identified modal parameters more precise.
\end{abstract}

\section{Introduction}

Under the effects of outburst accidents (such as earthquakes and wind) and operating loads, engineering structures can accumulate damage, which may lead to destructive accidents. In fact, internal damage of engineering structures inevitably leads to changes in structural dynamic parameters, such as the natural frequency, damping, and mode shapes. Therefore, structural modal identification has become the core technology of modern dynamic testing and on-line monitoring of complex engineering structures.

Modal identification and damage diagnosis based on structural vibrations have been widely adopted in many fields of civil engineering, but the application of this technology to hydraulic structures is still in the initial stage. In the 1960s, the construction bureau of California firstly developed the mechanical vibration machine and conducted an operational dynamic test on the Montst arch dam. The first four natural frequencies of the dam were successfully measured. In the 1990s, Houqun et al. [1] conducted prototype dynamic tests on the Dongjiang arch dam in Hunan Province and on the Longyangxia arch dam in Qinghai Province using a blasting method and extracted the corresponding modal parameters from the test results. Yifeng and Ming [2] established a weighted rubber model of a double-curvature arch dam. Jijian et al. [3] built a large-scale hydroelastic model of the Laxiwa arch dam to conduct the experimental modal analysis in order to identify the structural modal parameters. Mridha and Maity [4] investigated the nonlinear response of a concrete gravity dam-reservoir system using laboratory experiments on a small-scale model of the Koyna dam. Altunişik et al. [5] studied the variations of the modal parameters of a damaged arch dam before and after retrofitting using laboratory model experiments. Darbre et al. [6] researched 
the relationship between the dam natural frequency and reservoir water level using a plaster model of an arch dam. Loh and $\mathrm{Wu}[7]$ identified the modal parameters of the FeiTsui arch dam from monitoring data during a strong earthquake and studied the influence of the reservoir water level on the structural modal parameters and nonuniform input on the dynamic response of the arch dam. Mau and Wang [8] performed a system identification of an arch dam using vibrational test data. Sevim et al. [9] identified the modal parameters of the Berke arch dam using the frequency domain method based on the environmental excitations and then calibrated a three-dimensional FEM model with the results.

During modal experiments, determining the optimal number of sensors and corresponding configuration on structures has received increasing attention because inappropriate sensor placement can reduce the accuracy of the identified modal parameters. Meanwhile, modern concrete arch dams with complex structures and giant volumes may contain considerable degrees of freedom (DOFs); thus, the arrangement of sensors would particularly influence the measurement accuracy of the modal parameters [10,11]. During traditional dynamic tests of dams, the sensors are arranged based on engineering experience, which does not guarantee optimal sensor placement [12-14]. Therefore, research of the optimal sensor placement on concrete arch dams has become an important subject in recent years.

Many researchers have studied optimization criteria of sensor placement for structural modal identification in the past few years. In 1990, Kammer [15] proposed the effective independence (EFI) method to obtain the greatest spatial resolution of the targeted modes. The Fisher information matrix (FIM) is established to guarantee linear independence of interesting modal vectors and reserve the DOFs that contribute most to the independence of the targeted modes. Liu and Tasker [16] proposed the Multiple-Reference Ibrahim Time Domain method for sensor placement and developed a relationship between the sensor locations and variance of identification. By combining the Eigensystem Realization algorithm, Lim [17] arranged sensors to minimize the condition number of the Hankel matrix to maintain the independence of the targeted modes. Rafajłowicz [18] revealed a relationship between the information matrix and density of the input spectrum and measurement positions and studied the problem of sensor placement for parameter identification in the frequency domain. Xing and Bainum [19] researched the problem of optimal sensor placement based on the degree of controllability and observability of the discrete system. Reynier and Abou-Kandil [20] placed sensors by maximizing the minimum eigenvalue of the Gramian matrix. Shih et al. [21] defined the degree of controllability and observability as a second-order ordinary differential equation and deduced a relationship between the equation and frequency-response function. The sensors are arranged based on the contribution of each DOF to the index. Salama et al. [22] claimed that the sensors should be placed on the locations with great energy because it is good for modal identification and optimized the sensor locations by maximizing the modal strain energy (MSE). Baruh and Choe [23] used spline interpolation of the response of the measured points to obtain information about the unmeasured points. The sensors were optimized by minimizing the fitting error of interpolation. Breitfeld [24] arranged sensors by minimizing the off-diagonal elements of the Modal Assurance Criterion (MAC) matrix. Cruz et al. [25] established the fitness function by maximizing the natural frequency identification effectiveness and the mode shape independence and adopted the custom genetic algorithm to optimize the sensor configuration. Debnath et al. [26] evaluated the modal participation at individual degree of freedom (DOF) for the target modes and proposed the modal contribution in output energy (MCOE) as the optimization criterion. Papadimitriou [27] adopted the theory of information entropy to measure the uncertainty in the system parameters and proposed the nominal structural model to optimize the sensor configuration on the truss structure. In recent years, various optimization methods have been used in the process of sensor placement, such as the serial method [28, 29], particle swarm optimization [30], simulated annealing algorithm [31], and genetic algorithm [32].

This paper studied two traditional methods for maximizing the modal information, the effective independence method based on the maximal determinant of the FIM and the Kinetic Energy method based on the maximum modal strain energy. One drawback of the EFI method is that the locations with low modal stress energy may be selected, which would result in the loss of modal information. One drawback of the Kinetic Energy method is that the optimization process is highly dependent on the partitioning of the finite element mesh. Based on the above analysis, a novel optimization method combining the EFI method with the MSE method is proposed that could effectively resolve the drawbacks of the two methods. Considering the enormous number of DOFs of the hydraulic structure, a quantum genetic algorithm (QGA) is adopted to increase the computational efficiency and accuracy in this paper. Finally, a specific concrete arch dam is used as an example, and the sensors on the upstream surface are optimized with the proposed method. A comparison of the traditional and proposed methods shows that the proposed method has a higher convergence speed and better optimization accuracy, which has theoretical and practical application values.

\section{The Basic Principle of the Optimal Sensor Placement}

2.1. The Effective Independence Method. In 1991, Kammer presented the effective independence method and introduced the Fisher information matrix, which is based on the displacement modal matrix. To make the concerned modal vectors linearly independent, the measured points are ranked based on an effective independence value of the modal matrix and the measurement point with the minimum contribution is deleted in succession. For modal experiments that only identify the structural mode shapes, the generalized coordinates $q$ of the mode shapes can represent the identified parameters. The output value of the sensor can be expressed as follows:

$$
U_{s}=\Phi_{s} q
$$


where $U_{s}$ represents the output value of the sensor; $\Phi_{s}$ is the modal matrix; and $q$ is the vector of the modal coordinates.

The least square estimation of $q$ can be written as follows:

$$
\widehat{q}=\left[\Phi_{s}^{T} \Phi_{s}\right]^{-1} \Phi_{s} U_{s}
$$

By introducing measurement noise, (3) is defined as

$$
U_{s}=\Phi_{s} q+T
$$

where $T$ is the Gaussian white noise of a uniform distribution with the variance of $\psi^{2}$.

By minimizing the covariance matrix of the modal coordinates, the effective unbiased estimate of $q$ can be obtained as follows:

$$
P=E\left[(q-\widehat{q})(q-\hat{q})^{T}\right]=\left[\frac{1}{\psi^{2}} \Phi_{s}^{T} \Phi_{s}\right]^{-1}=\frac{1}{\psi^{2}} Q^{-1},
$$

where $Q=\Phi_{s}^{T} \Phi_{s}$ is the Fisher information matrix (FIM).

Therefore, an optimal estimation of the modal coordinates $q$ can be obtained when the determinant of the FIM is maximized. Then, the covariance matrix $P$ is minimized and the targeted modal vectors are linearly independent, which is good for structural modal identification.

Then, the corresponding fitness value $f_{1}$ of the EFI method can be represented as follows:

$$
f_{1}=\operatorname{det}(Q)=\operatorname{det}\left(\Phi_{s}^{T} \Phi_{s}\right)
$$

2.2. The Modal Strain Energy Method. Modal strain energy (MSE) is an index that is sensitive to the variations of the structural parameters and the ratio between the element MSE and total structural kinetic energy and is one of the system eigenvalues. Therefore, the MSE index is usually applied in structural damage identification.

The element MSE is defined as follows:

$$
\operatorname{MSE}_{i j}=\left\{\phi_{i}\right\}^{T}\left[k_{j}\right]\left\{\phi_{i}\right\},
$$

where $\left\{\phi_{i}\right\}$ is the $i$ th normalized mode shape and $\left[k_{j}\right]$ is the element stiffness matrix of the $j$ th element.

Similarly, the $i$ th structural modal strain energy can be expressed as follows:

$$
\mathrm{MSE}_{i}=\left\{\phi_{i}\right\}^{T}[K]\left\{\phi_{i}\right\},
$$

where $[K]$ is the global stiffness matrix of the structure.

This paper defines the fitness value of the optimal sensor placement based on the MSE as follows:

$$
f_{2}=\sum_{i=1}^{p} \sum_{j=1}^{p} \sum_{r \in q} \sum_{s \in q}\left|\phi_{r i} k_{r s} \phi_{s j}\right|
$$

where $k_{r s}$ represents the stiffness coefficient between the $r$ th DOF and $s$ th DOF; $\phi_{r i}$ is the deformation of the $r$ th element in the $i$ th mode; $\phi_{s j}$ is the deformation of the $s$ th element in the $j$ th mode; and $p$ and $q$ are the numbers of modes and measurement points, respectively.
2.3. The Combined Optimization Algorithm. Although the EFI method can make the selected modal vectors approximately linearly independent, the measured MSE of the selected points may be low, which would result in the loss of modal information. Because of this effect, this paper combines the EFI method with the MSE method and defines the fitness value $f_{3}$ as follows:

$$
f_{3}=f_{1} f_{2}=\alpha \operatorname{det}\left(\Phi_{s}^{T} \Phi_{s}\right) \sum_{i=1}^{p} \sum_{j=1}^{p} \sum_{r \in q} \sum_{s \in q}\left|\beta_{r i} k_{r s} \beta_{s j}\right|,
$$

where $\alpha$ is the adjustment parameter that scales the fitness value into the appropriate range.

\section{The Basic Theory of the Quantum Genetic Algorithm}

The quantum genetic algorithm (QGA) is a recently developed probability evolutionary algorithm that combines quantum computing with a genetic algorithm $[33,34]$. Based on quantum theory, the quantum probability vector is used to encode chromosomes, and the population is updated and optimized by adopting quantum-rotating doors to search for a globally optimal solution.

The smallest unit of information in the QGA is called a quantum bit and the state of a quantum bit can be expressed as follows:

$$
|\varphi\rangle=\alpha|0\rangle+\beta|1\rangle,
$$

where $\alpha, \beta$ represent the probability amplitudes of the quantum bit and meet the following normalized condition:

$$
|\alpha|^{2}+|\beta|^{2}=1
$$
follows:

Thus, the state of a quantum bit can also be written as

$$
|\varphi\rangle=\cos \frac{\theta}{2}|0\rangle+e^{i \varphi} \sin \frac{\theta}{2}|1\rangle .
$$

In the quantum genetic algorithm, quantum information is encoded by pairs of complex numbers. The quantum chromosome composed of $m$ sets of quantum bits can be written as follows:

$$
\left[\begin{array}{l|l|l|l}
\alpha_{1} & \alpha_{2} & \cdots & \alpha_{m} \\
\beta_{1} & \beta_{2} & \cdots & \beta_{m}
\end{array}\right]
$$

where $\left|\alpha_{i}\right|^{2}+\left|\beta_{i}\right|^{2}=1(i=1,2, \ldots, m)$.

This method can represent the random linear superposition of quantum states. For example, a chromosome with 3 quantum bits can be expressed as follows:

$$
\left[\begin{array}{c|c|c|}
\frac{1}{\sqrt{2}} & \frac{\sqrt{3}}{2} & \frac{1}{2} \\
\frac{1}{\sqrt{2}} & \frac{1}{2} & \frac{\sqrt{3}}{2}
\end{array}\right]
$$


An update of a quantum gate can be represented as

$$
\left[\begin{array}{c}
\alpha_{i}^{\prime} \\
\beta_{i}^{\prime}
\end{array}\right]=U\left(\theta_{i}\right)\left[\begin{array}{l}
\alpha_{i} \\
\beta_{i}
\end{array}\right]=\left[\begin{array}{cc}
\cos \left(\theta_{i}\right) & -\sin \left(\theta_{i}\right) \\
\sin \left(\theta_{i}\right) & \cos \left(\theta_{i}\right)
\end{array}\right]\left[\begin{array}{c}
\alpha_{i} \\
\beta_{i}
\end{array}\right],
$$

where $U\left(\theta_{i}\right)=\left[\begin{array}{cc}\cos \left(\theta_{i}\right) & -\sin \left(\theta_{i}\right) \\ \sin \left(\theta_{i}\right) & \cos \left(\theta_{i}\right)\end{array}\right]$ is the quantum-rotating gate, among which the variables are denoted as follows:

$$
\begin{aligned}
\theta_{i} & =k \cdot f\left(\alpha_{i}, \beta_{i}\right), \\
k & =\pi \cdot \exp \left(-\frac{t}{\text { iter }_{\text {max }}}\right),
\end{aligned}
$$

where $k$ is the adaptive variable; $t$ is the evolutionary population; and iter ${ }_{\max }$ is a constant that depends on the complexity of the optimization problem.

The search strategy of $f\left(\alpha_{i}, \beta_{i}\right)$ is shown in Table 1 . In Table $1, \alpha_{1}$ and $\beta_{1}$ are the probability amplitudes of the global optimal solutions, $d_{1}=\alpha_{1} \times \beta_{1}$ and $\xi_{1}=\tan ^{-1}\left(\beta_{1} / \alpha_{1}\right) . \alpha_{2}, \beta_{2}$ are the probability amplitude of the current solutions, $d_{2}=$ $\alpha_{2} \times \beta_{2}$ and $\xi_{2}=\tan ^{-1}\left(\beta_{2} / \alpha_{2}\right)$. If both $d_{1}$ and $d_{2}$ are greater than 0 , the current solutions and global optimal solutions will be in the first or third quadrant. When $\left|\xi_{1}\right|>\left|\xi_{2}\right|$, the current solution should be rotated counterclockwise, $f\left(\alpha_{i}, \beta_{i}\right)=+1$; otherwise, $f\left(\alpha_{i}, \beta_{i}\right)=-1$. The three other rotational criteria can be determined using the same method.

Furthermore, to prevent the optimization from converging at local extrema, the algorithm introduces a mutation operation based on a certain probability. For instance, a quantum bit $\alpha|0\rangle+\beta|1\rangle$ can be transferred into $\alpha|1\rangle+\beta|0\rangle$ through the operation. In the actual operation, the mutation probability is generally between 0.1 and 0.01 , which can both maintain the diversity of the population and prevent the algorithm from converging at local extremes.

The basic steps of the algorithm are shown in Figure 1.

\section{The Evaluation Criterion of Optimal Sensor Placement}

A good sensor network should allow the measured modal parameters to be sensitive to variations in the structural parameters and provide comprehensive information for structural damage diagnosis. In this paper, three criteria are selected to evaluate the ability of a sensor network to capture the structural dynamic response.

4.1. MAC. Based on orthogonality theory of modal vectors, the orthogonality of modal vectors directly influences the results of modal identification. Therefore, the MAC is selected as the comparison criterion of the optimal sensor placement as follows:

$$
\operatorname{MAC}_{i j}=\frac{\left(\Phi_{i} \Phi_{j}\right)^{2}}{\left(\Phi_{i}^{T} \Phi_{i}\right)\left(\Phi_{j}^{T} \Phi_{j}\right)}
$$

where $M A C_{i j}$ is the cosine value of the intersection angle between the $i$ th modal vector $\Phi_{i}$ and the $j$ th modal vector $\Phi_{j}$.
TABLE 1: The query table of $f\left(\alpha_{i}, \beta_{i}\right)$.

\begin{tabular}{lccc}
\hline$d_{1}$ & $d_{2}$ & \multicolumn{2}{c}{$f\left(\alpha_{i}, \beta_{i}\right)$} \\
\hline$d_{1}>0$ & $d_{2}>0$ & $\left|\xi_{1}\right|>\left|\xi_{2}\right|$ & $\left|\xi_{1}\right|<\left|\xi_{2}\right|$ \\
\hline True & True & +1 & -1 \\
True & False & +1 & +1 \\
False & True & -1 & -1 \\
False & False & -1 & +1 \\
\hline
\end{tabular}

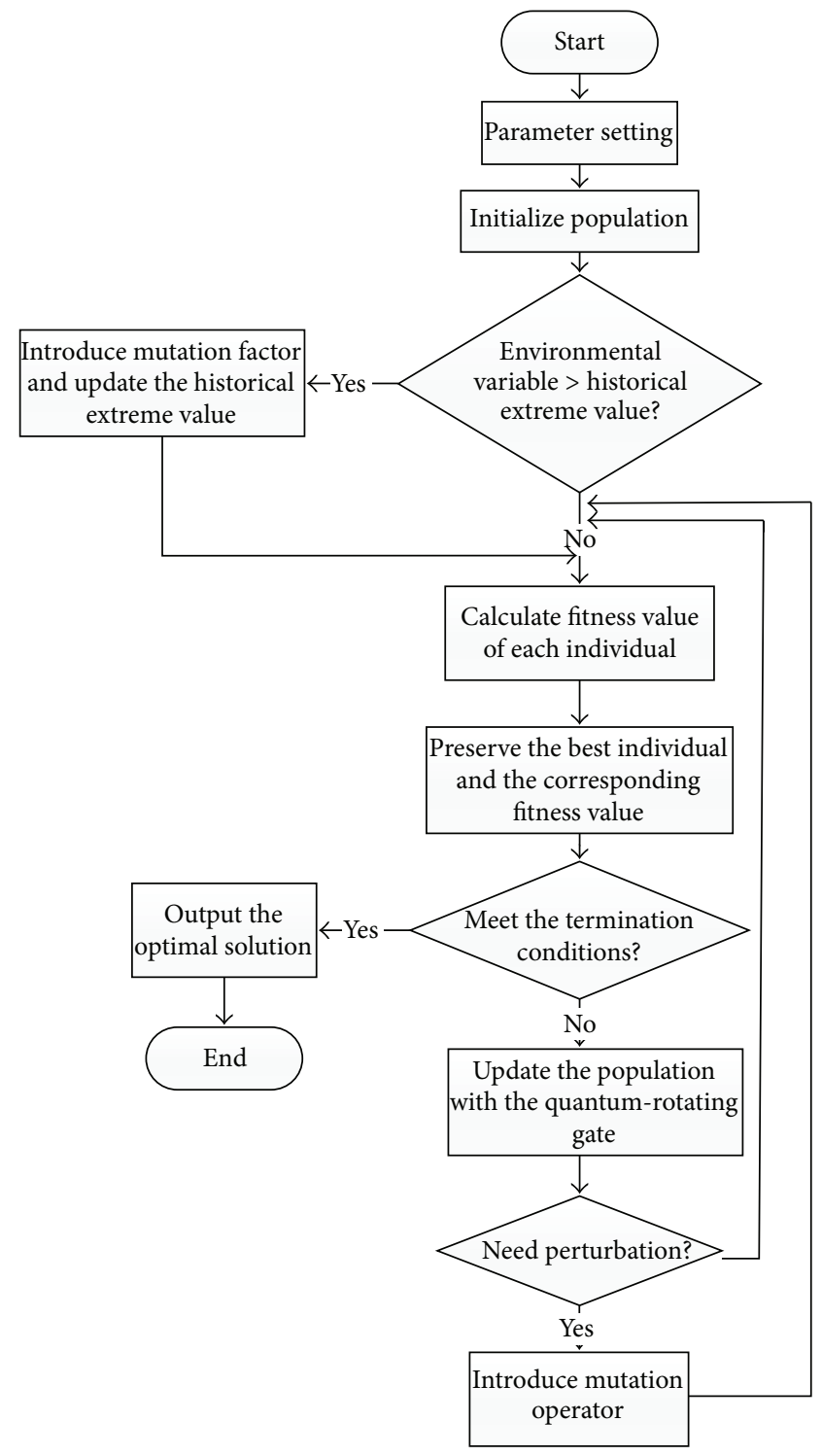

FIgURE 1: Flow chart of the quantum genetic algorithm.

The orthogonality of the modal vectors is better when the maximum value of the off-diagonal elements of the MAC matrix is smaller. Therefore, the maximum value of the offdiagonal elements of MAC matrix can be used as an index to evaluate the quality of the sensor placement scheme. 
4.2. The Criterion of Antinoise Performance. The identification of modal parameters is greatly influenced by ambient noise perturbations; under extreme conditions, the noise signal and structural vibration signal are not distinguishable. In this paper, the FIM determinant after the elimination of the DOFs of the candidate sensors is selected as the index for evaluating the modal identification ability of the sensor network under the effects of ambient noise perturbation.

4.3. The Criterion of the Condition Number. The condition number is a criterion for evaluating the morbidity of a matrix. Larger numbers mean that the modal matrix is more morbid and the identified parameters will be severely distorted. A modal matrix with a small condition number can perfectly resist ambient distortions. Therefore, the condition number of the modal matrix is used as a criterion for evaluating each optimizing method as is shown as follows:

$$
\operatorname{cond}(\Phi)=\|\Phi\| \cdot\left\|\Phi^{-1}\right\| \text {. }
$$

\section{Case Study}

5.1. Project Background. A double-curvature concrete arch dam is located midstream along the Lancang River in Yunnan Province. The elevation of the dam crest is $1,245 \mathrm{~m}$, with a maximum dam height of $292 \mathrm{~m}$ and a dam crest length of $922.74 \mathrm{~m}$. The top and bottom width of the crown cantilever are $13 \mathrm{~m}$ and $69.49 \mathrm{~m}$, respectively. The normal storage water level of the reservoir is $1,240 \mathrm{~m}$, with a total storage of 15.12 billion $\mathrm{m}^{3}$ and an effective storage of 9.895 billion $\mathrm{m}^{3}$, which makes it a pluriennial-regulation reservoir. The downstream elevation of the arch dam is shown in Figure 2.

A three-dimensional finite element model is developed to conduct vibration modal analysis of the dam-foundation system, as shown in Figure 3. The total number of elements and nodes of the model are 201,689 and 207,714, respectively. The model coordinates follow the right-hand screw rule. The direction of each axis is as follows: the $x$-axis is perpendicular to the flow direction and the left-bank direction is positive; the $y$-axis is parallel to the flow direction and the downstream direction is positive; the $z$-axis is parallel to the height direction and the downward direction is positive.

5.2. Modal Analysis. For large-scale civil structures, usually the displacements of low frequency modes could give sufficient information to describe the dynamic behavior of a structural system with sufficient accuracy to allow its health state modifications to be determined effectively [35]. It has been illustrated by [36] that the first ten mode shapes are able to reflect most of the dynamic characteristics of huge engineering structures. In recent years, many scholars have adopted no more than first ten mode shapes to study the structural dynamic behavior of the large-scale concrete dams, bridges and truss structures, and so forth [26, 37-39]. Therefore, in this paper, the first ten mode shapes of the dam are selected as the targeted modes. ABAQUS finite element software is used to conduct a dynamic modal analysis of the dam. Added-mass elements [40] are used to simulate the function of the upstream reservoir water. The first ten mode

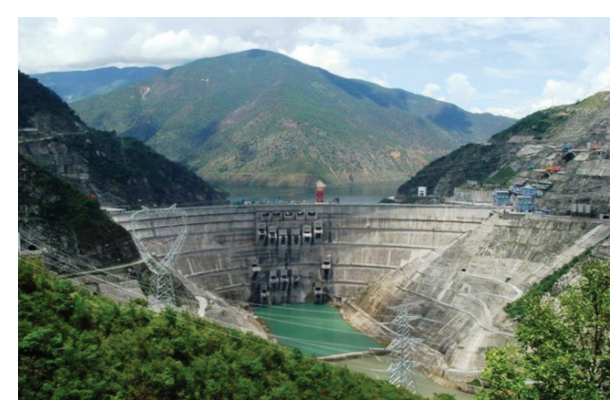

Figure 2: Downstream elevation of the arch dam.

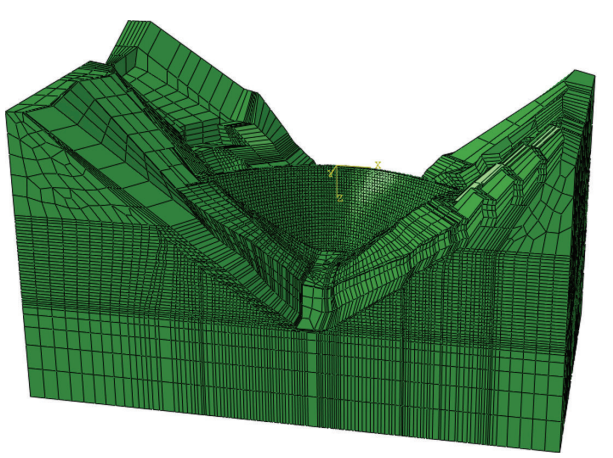

FIgure 3: Three-dimensional finite element model of the damfoundation system.

shapes and corresponding natural frequencies are shown in Figure 4 and Table 2, respectively.

5.3. Optimal Sensor Placement. There are 1,718 nodes on the upstream surface of the finite element model of the arch dam. In this paper, two DOFs ( $x$ direction and $y$ direction) of each node are considered. After eliminating 57 nodes that are unsuitable for sensor installation, there are 1,681 candidate nodes and 3,362 corresponding DOFs on the upstream surface. The quantum genetic algorithm is adopted to arrange 30 sets of sensors on the upstream surface based on the three criteria described previously.

The parameters of the quantum genetic algorithm are defined as follows: the initial population size and quantum bit are set as 3,000 and 15, respectively; the initial search is superposed based on an equal probability; and the corresponding probability amplitude is defined as $\pm \sqrt{2} / 2$. To verify the computation efficiency and quality of the quantum genetic algorithm, a traditional genetic algorithm (GA) is also used. The parameters of GA are listed as follows: the initial population size is 3000 ; the crossover rate and the mutation rate are defined as 0.75 and 0.02 , respectively. The maximum operating generations of the two algorithms are defined as 2000. Evolution lines of the two methods for different optimization criteria are shown in Figure 5.

It is clear that the QGA can always converge to a higher fitness value in fewer generations than the GA. The premature phenomenon of the GA can be effectively avoided by adopting QGA. 


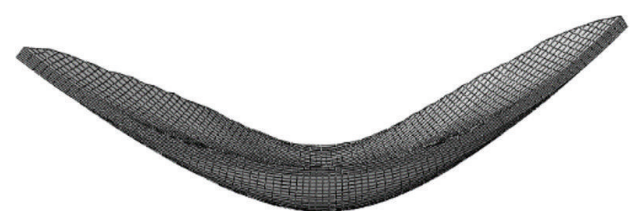

(a) Mode 1

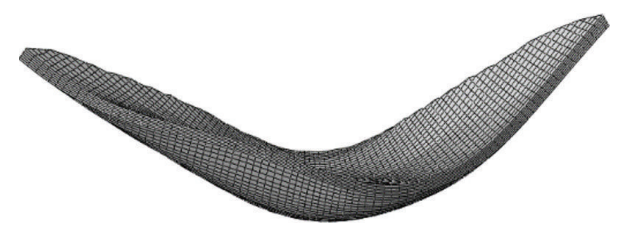

(c) Mode 5

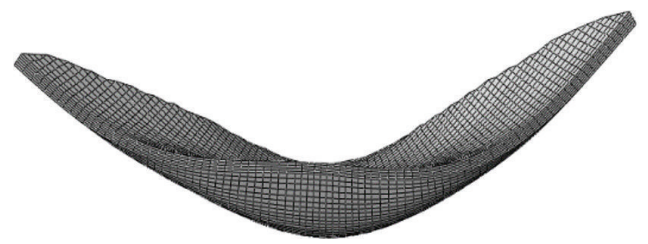

(e) Mode 9

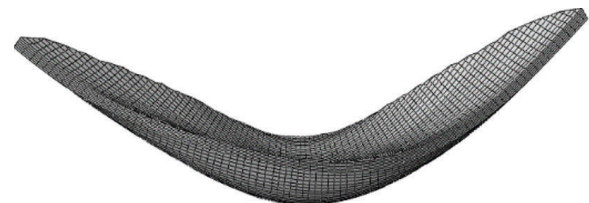

(b) Mode 3

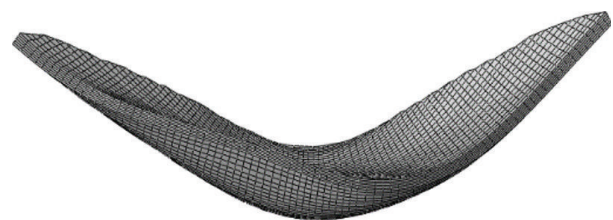

(d) Mode 7

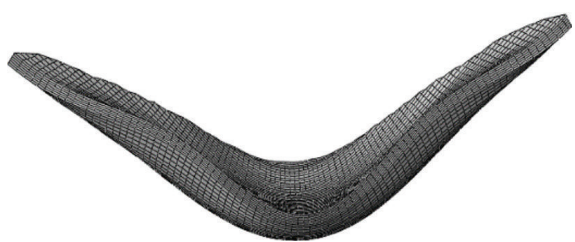

(f) Mode 10

Figure 4: Typical vibration modes of the arch dam.

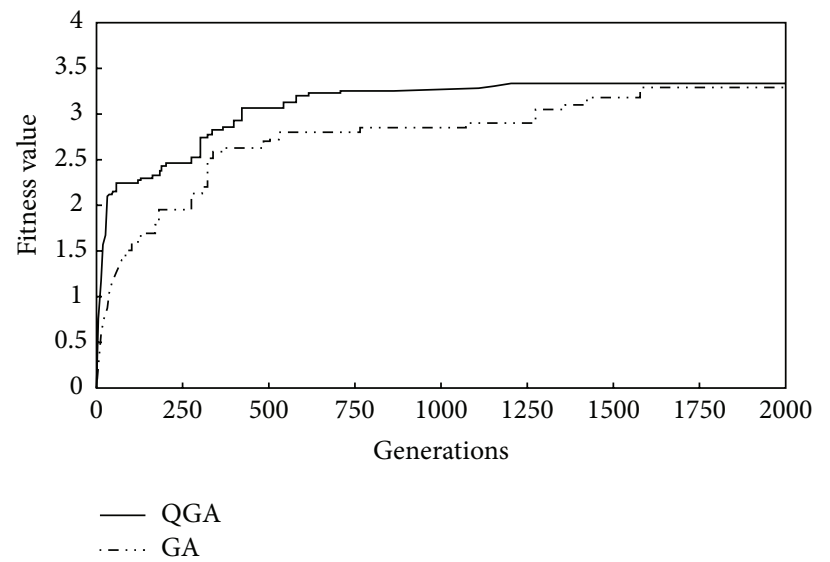

(a) The evolution line of fitness value $f_{1}$

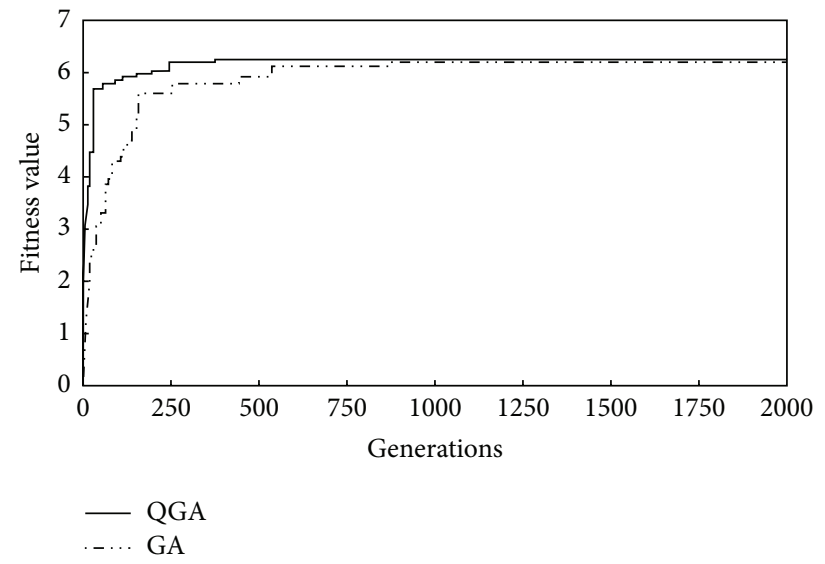

(b) The evolution line of fitness value $f_{2}$

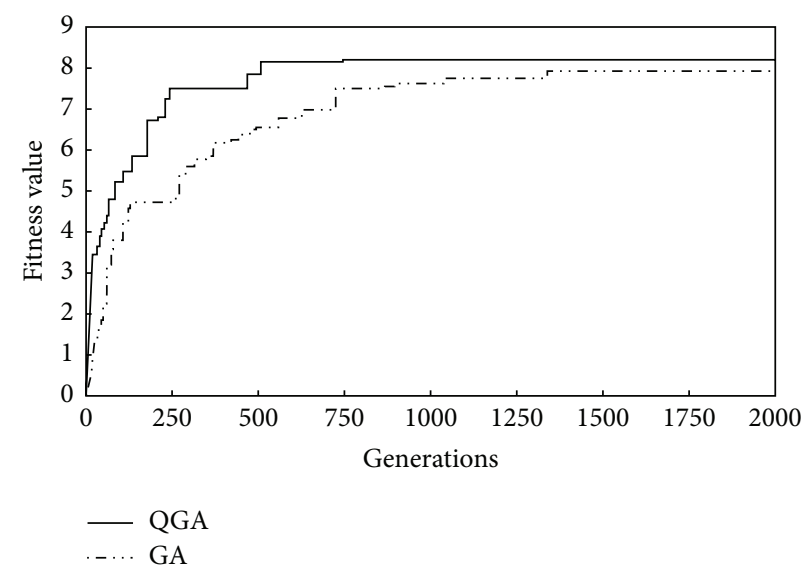

(c) The evolution line of fitness value $f_{3}$

Figure 5: The evolution line of fitness value using the QGA and GA. 
TABLE 2: The natural frequency of the arch dam.

\begin{tabular}{lcccccccccc}
\hline Mode & 1 & 2 & 3 & 4 & 5 & 6 & 7 & 8 & 9 & 10 \\
\hline Frequency & 1.327 & 1.833 & 2.481 & 2.828 & 3.148 & 3.859 & 4.023 & 4.618 & 5.153 & 5.359 \\
\hline
\end{tabular}

TABLE 3: The statistic of the fitness value after multiple runs based on $f_{1}$ criterion.

\begin{tabular}{lccccccccccc}
\hline Algorithm & Number of runs & 20 & 50 & 100 & 200 & 300 & 400 & 500 & 1000 & 1500 & 2000 \\
\hline \multirow{2}{*}{ QGA } & Average value & 0.88 & 1.43 & 1.69 & 2.08 & 2.23 & 2.41 & 2.54 & 2.91 & 3.05 & 3.12 \\
& Best value & 1.57 & 2.15 & 2.24 & 2.43 & 2.52 & 2.93 & 3.06 & 3.25 & 3.34 & 3.34 \\
\hline \multirow{2}{*}{ GA } & Average value & 0.45 & 0.73 & 0.98 & 1.36 & 1.62 & 1.87 & 2.04 & 2.78 & 2.91 & 3.01 \\
& Best value & 0.75 & 1.15 & 1.51 & 1.95 & 2.13 & 2.63 & 2.70 & 2.85 & 3.18 & 3.29 \\
\hline
\end{tabular}

TABLE 4: The statistic of the fitness value after multiple runs based on $f_{2}$ criterion.

\begin{tabular}{lccccccccccc}
\hline Algorithm & Number of runs & 20 & 50 & 100 & 200 & 300 & 400 & 500 & 1000 & 1500 & 2000 \\
\hline \multirow{2}{*}{ QGA } & Average value & 2.25 & 3.65 & 4.14 & 4.72 & 4.82 & 5.18 & 5.39 & 5.82 & 5.96 & 6.03 \\
& Best value & 4.37 & 5.68 & 5.96 & 6.03 & 6.2 & 6.25 & 6.25 & 6.25 & 6.25 & 6.25 \\
\hline \multirow{2}{*}{ GA } & Average value & 1.88 & 2.21 & 2.84 & 3.59 & 3.71 & 4.23 & 4.57 & 5.39 & 5.66 & 5.80 \\
& Best value & 2.48 & 3.06 & 4.30 & 5.60 & 5.79 & 5.79 & 5.92 & 6.20 & 6.20 & 6.20 \\
\hline
\end{tabular}

TABLE 5: The statistic of the fitness value after multiple runs based on $f_{3}$ criterion.

\begin{tabular}{lccccccccccc}
\hline Algorithm & Number of runs & 20 & 50 & 100 & 200 & 300 & 400 & 500 & 1000 & 1500 & 2000 \\
\hline \multirow{2}{*}{ QGA } & Average value & 1.79 & 3.11 & 3.77 & 4.29 & 5.36 & 5.90 & 6.29 & 7.25 & 7.57 & 7.73 \\
& Best value & 3.45 & 4.08 & 5.23 & 6.73 & 7.50 & 7.50 & 7.85 & 8.20 & 8.2 & 8.2 \\
\hline \multirow{2}{*}{ GA } & Average value & 0.47 & 1.36 & 2.14 & 2.78 & 3.72 & 4.34 & 4.78 & 6.21 & 6.78 & 7.07 \\
& Best value & 0.85 & 2.25 & 3.80 & 4.73 & 5.60 & 6.18 & 6.55 & 7.63 & 7.93 & 7.93 \\
\hline
\end{tabular}

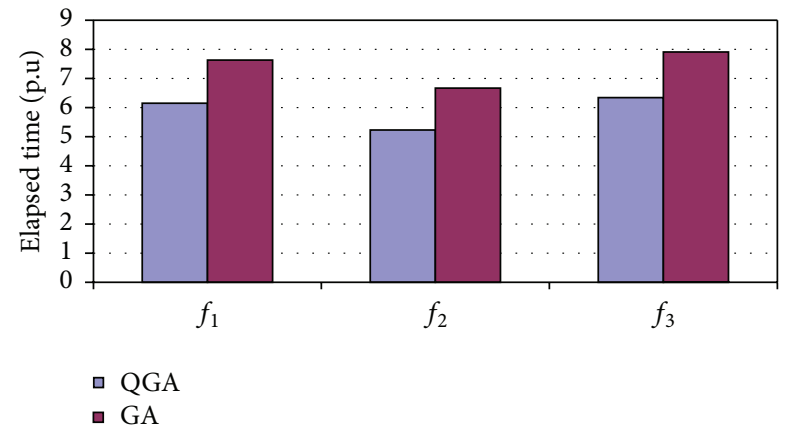

FIGURE 6: The comparison of total elapsed time between QGA and GA.

The statistic of multiple runs of the QGA and GA based on three criteria is shown in Tables 3-5. As shown in the tables, the average fitness value and the best fitness value of the QGA out of different runs are all greater than those of the GA. The QGA possesses higher computation quality with the same operating generations comparing with the GA.

The total elapsed time of the two algorithms is shown in Figure 6. As is shown in figure, the total operating time of QGA based on three criteria is always shorter than the GA. Therefore, it can be concluded that the QGA possesses higher
TABle 6: The statistics of the off-diagonal elements of the MAC matrix based on three criteria.

\begin{tabular}{lccc}
\hline Optimal criterion & $\begin{array}{c}\text { Minimum } \\
\text { value }\end{array}$ & Average value & Maximum value \\
\hline$f_{1}$ & 0.005 & 0.019 & 0.032 \\
$f_{2}$ & 0.006 & 0.027 & 0.043 \\
$f_{3}$ & 0.003 & 0.007 & 0.04 \\
\hline
\end{tabular}

optimization accuracy and better computation efficiency comparing with the GA.

Bar graphs of the MAC matrix for the three criteria obtained by the quantum genetic algorithm are shown in Figure 7 and the relevant statistics of the off-diagonal elements in MAC matrixes are shown in Table 6. We can see that the average value and the maximum value of the offdiagonal elements obtained by the $f_{3}$ criterion are much less than those with the other two criteria. The off-diagonal element $\mathrm{MAC}_{i j}$ represents the crossing angle of the two corresponding modal vectors. According to [25], the smaller the off-diagonal elements of the MAC matrix are, the more independent the different modal vectors remain. Therefore, it can be concluded that the sensor configuration obtained by the $f_{3}$ criterion can improve the spatial intersection angle among the modal vectors and thus better represent the modal characteristics of the dam structure. 
TABLE 7: FIM determinant and the condition number of the vibration mode based on three criteria.

\begin{tabular}{lcc}
\hline The sensor placement scheme & The determinant of FIM & The condition number of the vibration mode \\
\hline$f_{1}$ & $1.64 E-15$ & 19 \\
$f_{2}$ & $7.54 E-16$ & 27 \\
$f_{3}$ & $3.21 E-13$ & 11 \\
\hline
\end{tabular}

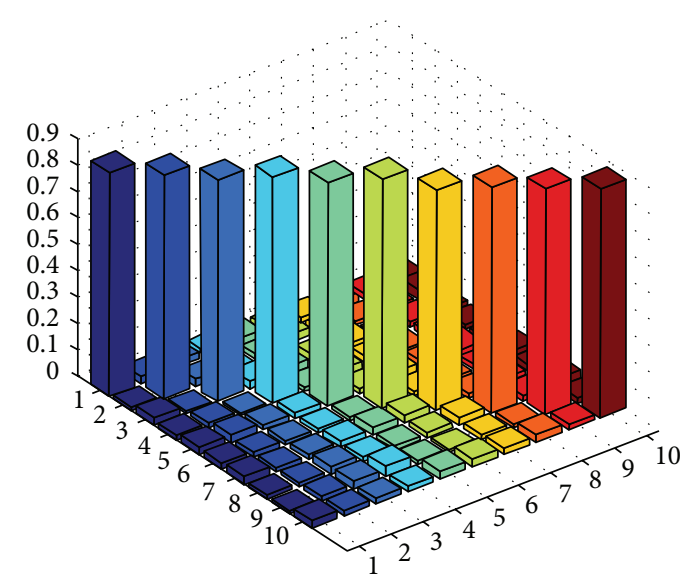

(a) The MAC matrix based on the $f_{1}$ criterion

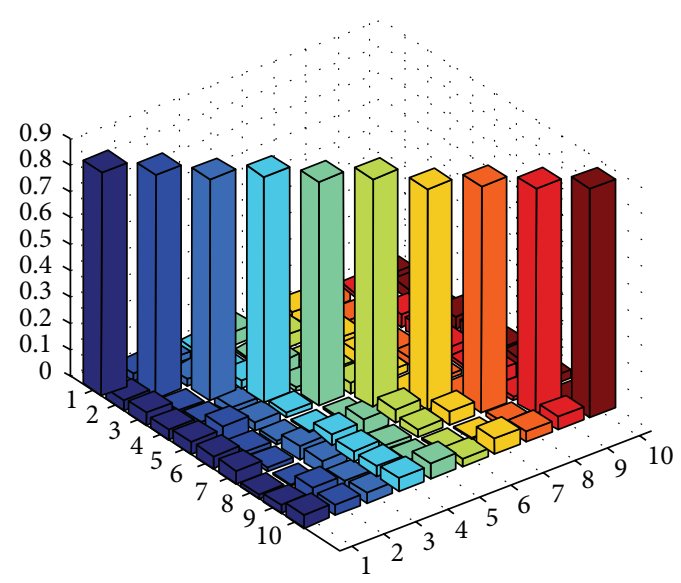

(b) The MAC matric based on the $f_{2}$ criterion

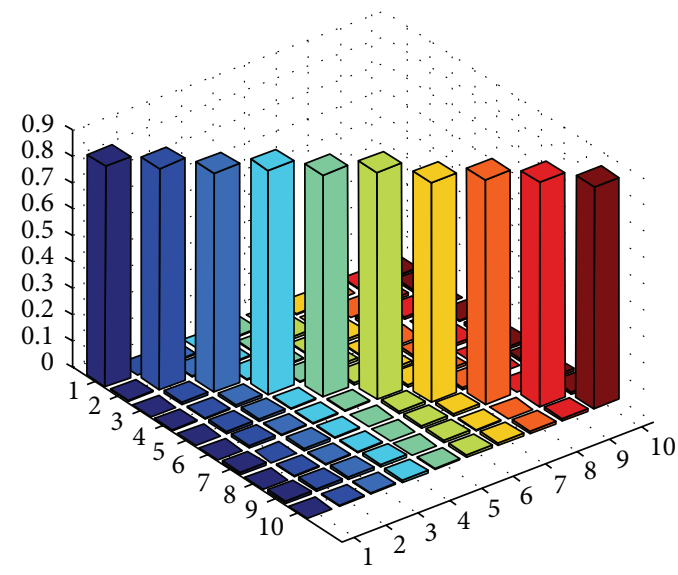

(c) The MAC matrix based on the $f_{3}$ criterion

FIGURE 7: The bar graph of the optimal MAC values based on different criteria.

The determinant of the FIM after removing the candidate sensors and condition number of the modal matrix are shown in Table 7 and the following conclusions could be drawn:

(1) The FIM determinant after eliminating the DOFs of the candidate sensors can be seen as the measure of the uniformity between the measured mode and the results of the FEM methods. It is the indicator which evaluates the influence of the ambient noise on the measured mode [26]. As is shown in Table 7, the obtained FIM determinant based on the $f_{3}$ criterion is far greater than those of the other two criteria. Therefore, it can be concluded that the sensor network obtained by the proposed method possesses a better antinoise property.
(2) According to [41], the matrix condition number represents the sensibility of matrix calculation on computation error. The larger the condition number, the more ill-conditioned the modal matrix, whereas a matrix with condition number close to 1 would make the identified modal characteristics more accurate [42]. As is shown in Table 7, the condition number obtained by the $f_{3}$ criterion is 11 , which is much less than those of the other two criteria (19 and 27). Therefore, it can be concluded that the proposed $f_{3}$ criterion can effectively resist the ambient environmental perturbation and raise the accuracy of the identified modal parameters.

In conclusion, the combined optimization criterion based on a quantum genetic algorithm is shown to be better than 


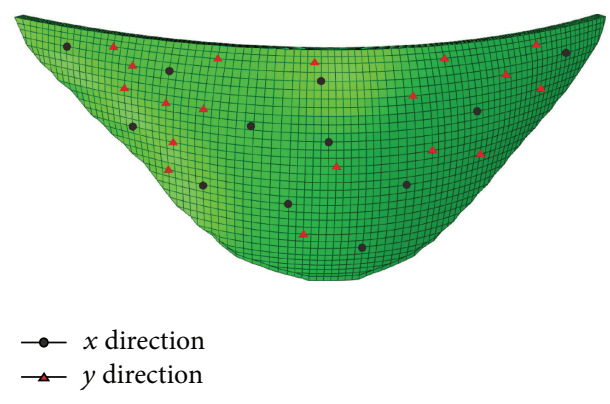

FIGURE 8: Final sensor network on the arch dam.

traditional methods. The final optimal sensor network is shown in Figure 8.

\section{Conclusion}

This paper proposes a novel optimization criterion for sensor placement by combining the effective independent method with the modal strain energy method to improve the modal identification ability. Moreover, to increase the computing efficiency and accuracy, a quantum genetic algorithm is adopted. By comparing the traditional methods, the following conclusions could be drawn:

(1) By combining the quantum computing principle with a genetic algorithm, the QGA adopts the vector of a quantum state to represent the genetic encoding and updates the chromosomes with a quantum-rotating gate to improve the efficiency of the optimization. In comparison with a traditional genetic algorithm, it is clear that the algorithm possesses the property of global convergence and always converges to a higher fitness value in fewer generations than the GA.

(2) The off-diagonal elements of the MAC matrix obtained using the combined optimization criterion are much smaller than those obtained using traditional methods. It is verified that the sensor network obtained using the proposed method can maximize the spatial intersection angle among different modal vectors, which could increase the modal identification accuracy.

(3) The determinant of the FIM obtained with the proposed method is obviously greater than that obtained using traditional methods, while the condition number of the modal matrix is much lower than those obtained using the traditional methods. The obtained sensor network can effectively resist ambient perturbations, which also increase the modal identification accuracy.

\section{Conflict of Interests}

The authors declare that there is no conflict of interests regarding the publication of this paper.

\section{References}

[1] C. Houqun, H. Shunzai, and S. Kezhong, The Field Test Report of Dynamic Interaction of Dam-Foundation-Water of Dongjiang Arch Dam, China Institute of Water Resources and Hydropower Research, Beijing, China, 1993.

[2] W. Yifeng and G. Ming, "The experimental modal analysis of arch dam," in Hydro-Science and Engineering, pp. 857-860, 1991.

[3] L. Jijian, Z. Jianwei, and L. Huokun, "Study on modal parameter identification of high arch dam under flood discharge excitation," Journal of Vibration and Shock, vol. 26, no. 12, pp. 101-105, 2007.

[4] S. Mridha and D. Maity, "Experimental investigation on nonlinear dynamic response of concrete gravity dam-reservoir system," Engineering Structures, vol. 80, pp. 289-297, 2014.

[5] A. C. A. Altunişik, M. Günaydin, B. Sevim, A. Bayraktar, and S. Adanur, "CFRP composite retrofitting effect on the dynamic characteristics of arch dams," Soil Dynamics \& Earthquake Engineering, vol. 74, pp. 1-9, 2015.

[6] G. R. Darbre, C. A. M. De Smet, and C. Kraemer, "Natural frequencies measured from ambient vibration response of the arch dam of Mauvoisin," Earthquake Engineering and Structural Dynamics, vol. 29, no. 5, pp. 577-586, 2000.

[7] C.-H. Loh and T.-S. Wu, "Identification of Fei-Tsui arch dam from both ambient and seismic response data," Soil Dynamics \& Earthquake Engineering, vol. 15, no. 7, pp. 465-483, 1996.

[8] S. T. Mau and S. Wang, "Arch dam system identification using vibration test data," Earthquake Engineering \& Structural Dynamics, vol. 18, no. 4, pp. 491-505, 1989.

[9] B. Sevim, A. C. Altunişik, and A. Bayraktar, "Earthquake behavior of berke arch dam using ambient vibration test results," Journal of Performance of Constructed Facilities, vol. 26, no. 6, pp. 780-792, 2012.

[10] I. Matiss, "Multi-element capacitive sensor for non-destructive measurement of the dielectric permittivity and thickness of dielectric plates and shells," NDT and E International, vol. 66, pp. 99-105, 2014.

[11] K. J. Lemler and W. H. Semke, "The reduction of modal sensor channels through a pareto chart methodology," Journal of Sensors, vol. 2015, Article ID 869258, 10 pages, 2015.

[12] A. Cunha, E. Caetano, and R. Delgado, "Dynamic tests on large cable-stayed bridge," Journal of Bridge Engineering, vol. 6, no. 1, pp. 54-62, 2001.

[13] A. K. Pandey, M. Biswas, and M. M. Samman, "Damage detection from changes in curvature mode shapes," Journal of Sound and Vibration, vol. 145, no. 2, pp. 321-332, 1991.

[14] M. G. Srinivasan and C. A. Kot, Effect of Damage on the Modal Parameters of a Cylindrical Shell, Argonne National Laboratory, Lemont, Ill, USA, 1992.

[15] D. C. Kammer, "Sensor placement for on-orbit modal identification and correlation of large space structures," in Proceedings of the American Control Conference, pp. 2984-2990, San Diego, Calif, USA, May 1990.

[16] C. Liu and F. A. Tasker, "Sensor placement for multi-input multi-output dynamic identification," in Proceedings of the 36th Structures, Structural Dynamics and Materials Conference (SDM '95), AIAA-95-1114-CP, New Orleans, La, USA, April 1995.

[17] T. W. Lim, "Actuator/sensor placement for modal parameter identification of flexible structures," The International Journal of Analytical \& Experimental Modal Analysis, vol. 8, no. 1, pp. 1-13, 1993. 
[18] E. Rafajłowicz, "Optimal experiment design for identification of linear distributed-parameter systems: frequency domain approach," IEEE Transactions on Automatic Control, vol. 28, no. 7, pp. 806-808, 1983.

[19] G.-Q. Xing and P. M. Bainum, "Actuator placement using degree of controllability for discrete-time systems," Control \& Dynamic Systems, vol. 79, pp. 51-88, 1996.

[20] M. Reynier and H. Abou-Kandil, "Sensors location for updating problems," Mechanical Systems and Signal Processing, vol. 13, no. 2, pp. 297-314, 1999.

[21] Y.-T. Shih, A. C. Lee, and J.-H. Chen, "Sensor and actuator placement for modal identification," Mechanical Systems and Signal Processing, vol. 12, no. 5, pp. 641-659, 1998.

[22] M. Salama, T. Rose, and J. Garba, "Optimal placement of excitations and sensors for verification of large dynamical systems," in Proceedings of the 28th Structures, Structural Dynamics and Materials Conference (SDM '87), K. T. Kedward, Ed., Monterey, Calif, USA, April 1987.

[23] H. Baruh and K. Choe, "Sensor-failure detection method for flexible structures," Journal of Guidance, Control, and Dynamics, vol. 10, no. 5, pp. 474-482, 1987.

[24] T. Breitfeld, "A method for identification of a set of optimal measurement points for experimental modal analysis," Modal Analysis the International Journal of Analytical \& Experimental Modal Analysis, vol. 11, no. 1-2, pp. 1-9, 1996.

[25] A. Cruz, W. Vélez, and P. Thomson, "Optimal sensor placement for modal identification of structures using genetic algorithms - a case study: the olympic stadium in Cali, Colombia," Annals of Operations Research, vol. 181, no. 1, pp. 769-781, 2010.

[26] N. Debnath, A. Dutta, and S. K. Deb, "Placement of sensors in operational modal analysis for truss bridges," Mechanical Systems \& Signal Processing, vol. 31, no. 8, pp. 196-216, 2012.

[27] C. Papadimitriou, "Optimal sensor placement methodology for parametric identification of structural systems," Journal of Sound and Vibration, vol. 278, no. 4-5, pp. 923-947, 2004.

[28] J. Liu and W. Huang, "Application of a cumulative method of sensor placement for offshore platforms," Journal of Ocean University of Qingdao, vol. 33, no. 3, pp. 476-482, 2003.

[29] J. Liu and W. P. Huang, "The reduced method of optimal sensor placement," Ocean Engineering, vol. 22, no. 1, pp. 32-13, 2004.

[30] X. K. Lin and B. Y. Qin, "Application of particle swarm optimization to optimal sensor placement," Control Engineering of China, vol. 20, no. 1, pp. 83-84, 2013.

[31] G. Rongxiong, G. Rong, and W. Liqiong, "Study on optimized layout of sensors based on simulated annealing algorithm using mac criterion," China Municipal Engineering, vol. 5, pp. 103-107, 2014.

[32] W. Liu, W.-C. Gao, Y. Sun, and M.-J. Xu, "Optimal sensor placement for spatial lattice structure based on genetic algorithms," Journal of Sound \& Vibration, vol. 317, no. 1-2, pp. 175-189, 2008.

[33] Z. Shu, P. Wei, Z. Wei-Li, and D. Ying, "A novel quantum genetic algorithm based on particle swarm optimization method and its application," Acta Electronica Sinica, vol. 34, no. 5, pp. 897-901, 2006.

[34] K.-H. Han and J.-H. Kim, "Genetic quantum algorithm and its application to combinatorial optimization problem," in Proceedings of the Congress on Evolutionary Computation, vol. 2, pp. 1354-1360, IEEE, La Jolla, Calif, USA, July 2000.

[35] F. Kang, J.-J. Li, and Q. Xu, "Virus coevolution parthenogenetic algorithms for optimal sensor placement," Advanced Engineering Informatics, vol. 22, no. 3, pp. 362-370, 2008.
[36] Y. Zhiyuan, The Research of the Theory and Method for the Modal Identification of Huge Engineering Structures, Southeast University, Nanjing, China, 2004.

[37] M. Meo and G. Zumpano, "On the optimal sensor placement techniques for a bridge structure," Engineering Structures, vol. 27, no. 10, pp. 1488-1497, 2005.

[38] V. Meruane and W. Heylen, "An hybrid real genetic algorithm to detect structural damage using modal properties," Mechanical Systems and Signal Processing, vol. 25, no. 5, pp. 1559-1573, 2011.

[39] S. V. Calcina, L. Eltrudis, L. Piroddi, and G. Ranieri, "Ambient vibration tests of an arch dam with different reservoir water levels: experimental results and comparison with finite element modelling," The Scientific World Journal, vol. 2014, Article ID 692709, 12 pages, 2014.

[40] H. M. Westergaard, "Water pressures on dams during earthquakes," Transactions of the American Society of Civil Engineers, vol. 98, no. 2, pp. 418-432, 1933.

[41] C. Rainieri and G. Fabbrocino, Operational Modal Analysis of Civil Engineering Structures, Springer, New York, NY, USA, 2014.

[42] A. Lazaridis, "A note regarding the condition number: the case of spurious and latent multicollinearity," Quality \& Quantity, vol. 41, no. 1, pp. 123-135, 2007. 


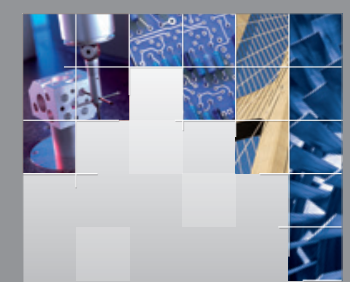

\section{Enfincering}
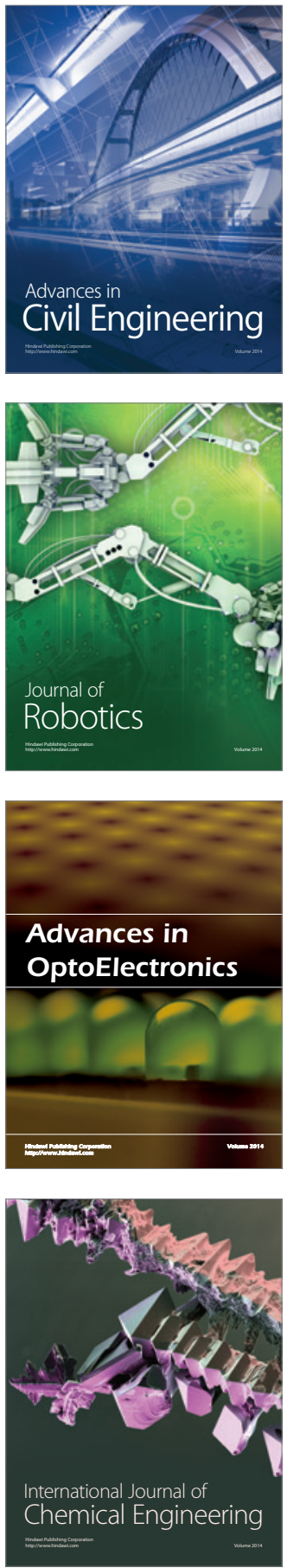

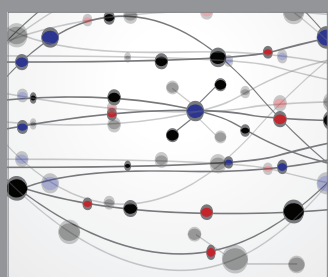

The Scientific World Journal

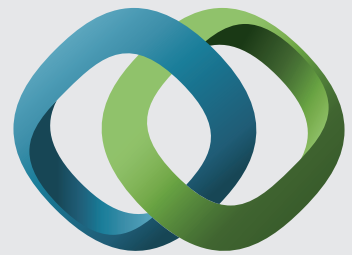

\section{Hindawi}

Submit your manuscripts at

http://www.hindawi.com
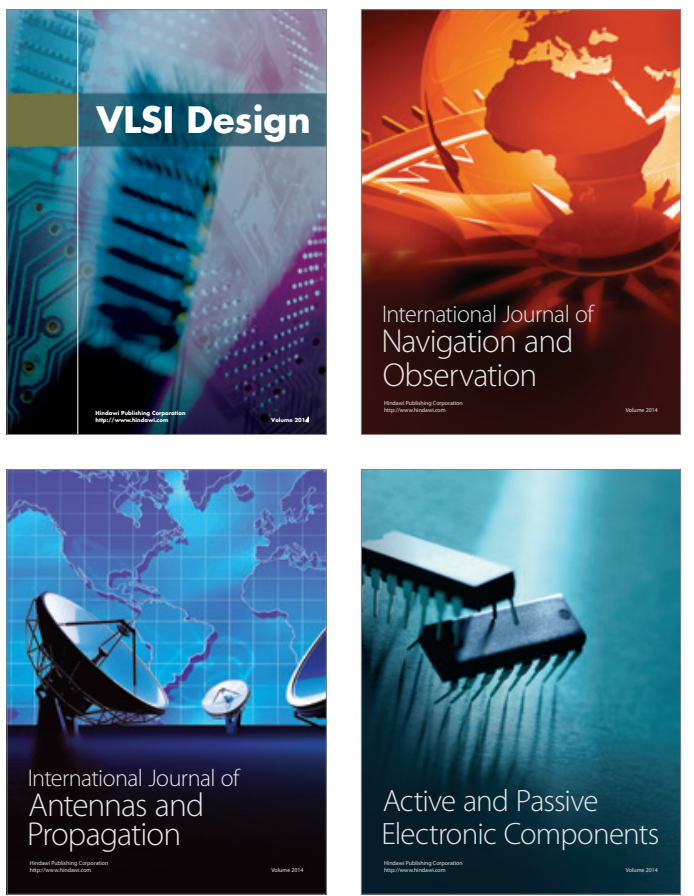
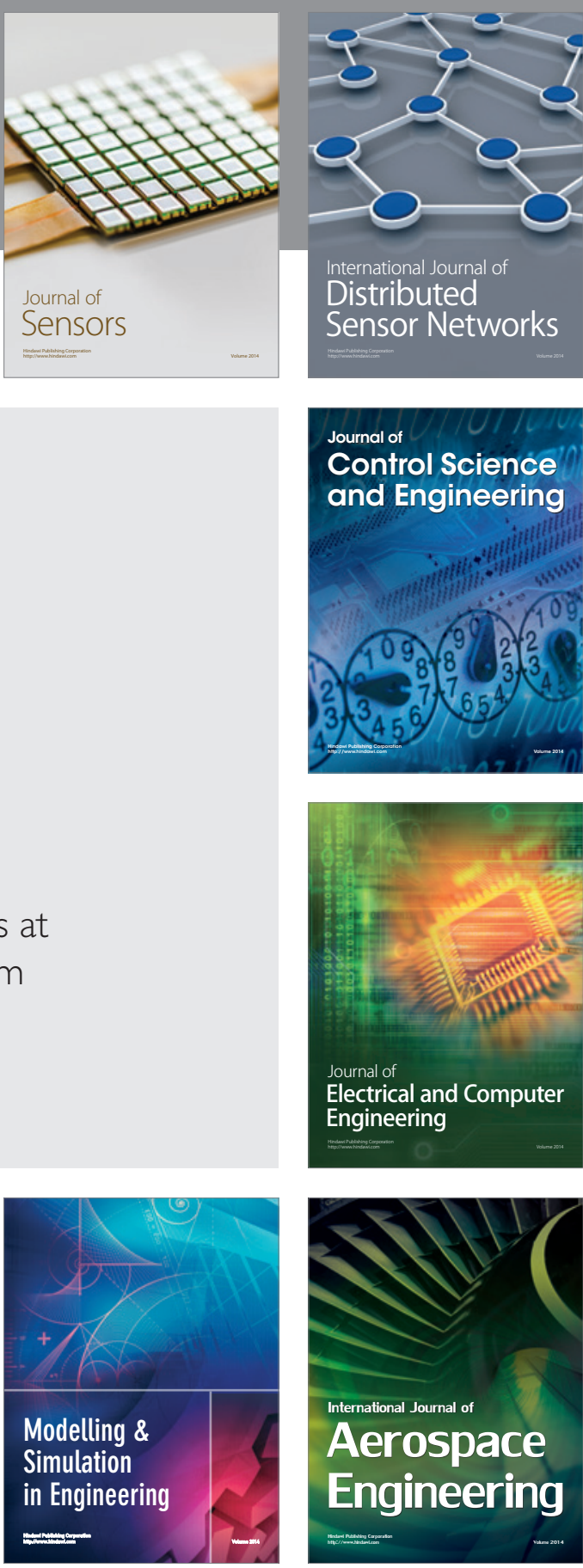

International Journal of

Distributed

Sensor Networks

Journal of

Control Science

and Engineering
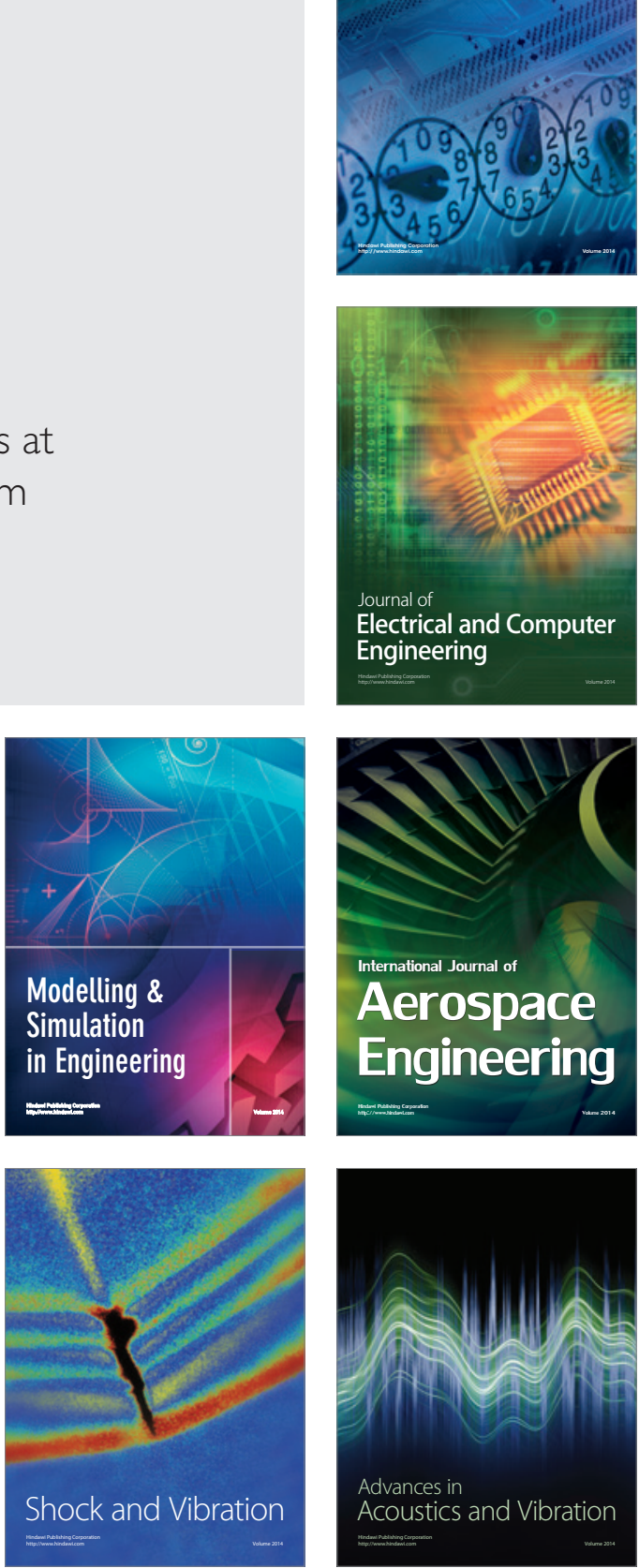